\title{
OPEN Chronic effects of high-intensity functional training on motor function: a systematic review with multilevel meta-analysis
}

\begin{abstract}
Jan Wilke ${ }^{\bowtie}$ Lisa Mohr
High-intensity functional training (HIFT) has become a popular method in the sports and fitness sector. In contrast to unimodal approaches such as strength or endurance training, it has been hypothesized to induce concurrent adaptations in multiple markers of motor function. However, to date, the effectiveness of HIFT in this regard has not been studied. The present systematic review quantified the chronic effects of HIFT on motor function in healthy individuals. A multilevel metaanalysis with a robust random effects meta-regession model was used to pool the standardized mean differences (SMD) between (a) HIFT and (b) no-exercise (NEX) as well as conventional endurance, resistance and balance training for outcomes of muscle strength, endurance capacity and balance. The influence of possible effect modifiers such as program duration, session duration, age or sex was examined in a moderator analysis. Seventeen papers with moderate to high methodological quality (PEDro scale) were identified. Compared to NEX, HIFT had small to moderate positive effects on endurance capacity (SMD: $0.42,95 \% \mathrm{Cl} 0.07-0.78, \mathrm{p}=0.03)$ and strength $(0.60,95 \% \mathrm{Cl} 0.02-1.18$, $\mathrm{p}=0.04$ ) but no effect on balance (SMD: $-0.10,95 \% \mathrm{Cl}-1.13$ to $0.92, \mathrm{p}=0.42$ ). Regarding endurance, HIFT showed similar effectiveness as moderate-intensity endurance training (SMD: $-0.11,95 \% \mathrm{CI}$ -1.17 to $0.95, \mathrm{p}=0.75$ ) and high-intensity interval endurance training (SMD: $-0.15,95 \% \mathrm{Cl}-1.4$ to $1.1, p=0.66$ ). No comparisons of HIFT vs. classical resistance or balance training were found. Moderator analyses revealed no influence of most effect modifiers. However, regarding endurance, females seemed to respond more strongly to HIFT in the comparison to NEX ( $<$ <.05). HIFT appears to represent an appropriate method to induce chronic improvements in motor function. While being superior to NEX and non-inferior to endurance training, current evidence does not allow a comparison against resistance and balance training. The impact of possible effect moderators should be further elucidated in future research.
\end{abstract}

High-intensity functional training (HIFT) has become a popular trend in the sports and fitness sector ${ }^{1}$. It is commonly characterized as the strenuous performance of exercises mimicking movements of daily life (e.g. squats, lunges or push-ups) interspersed with short breaks ${ }^{2}$. Contrary to high-intensity training, which is rather unidimensional and typically focused on one motor ability (e.g. running or cycling to improve endurance), HIFT aims to integrate cardiovascular, neuromotor and muscular efforts which is achieved by a variety of strategies such as the selection of whole-body exercises maximizing oxygen consumption, fast movement execution and the optional use of scalable weights (e.g. dumbbells, medicine balls, resistance bands).

HIFT may have relevant advantages for different populations. Many inactive individuals report a lack of time to represent a significant barrier to engaging in physical activity ${ }^{3,4}$. HIFT workouts tend to have short durations of mostly below $30 \mathrm{~min}$ and may thus be more appealing than conventional programs with longer durations ${ }^{2}$. In addition, stronger increases in intrinsic motivation predicting long-term activity adherence have been observed following HIFT when compared to continuous, moderate-intensity exercise ${ }^{5,6}$. While these data render HIFT an interesting option for sedentary individuals, it may also be of interest for athletes. Analyses of team sports show that many markers of motor function (e.g. strength, running endurance, postural control) are not or only weakly predictive of performance when considered in isolation ${ }^{7,8}$. This may be due to the fact that most sports 
do require a fine-orchestrated combination of motor skills. Owing to its multimodal nature, HIFT's ecological validity for sporting performance may be higher than that of traditional approaches.

In order to gauge the potential of HIFT in exercise counseling for inactive individuals and program design for athletes, its effectiveness needs to be compared to classical exercise regimes. Despite the existence of related trials with an appropriate study design (RCT and crossover trials), there is no quantitative data synthesis pooling their findings using meta-analytic techniques. Furthermore, it is unknown, which variables moderate the potential effects of HIFT on parameters of motor function. The present systematic review with meta-analysis, therefore aimed to investigate the effects of HIFT on motor performance as compared to classical training methods.

\section{Methods}

A systematic review with multilevel meta-analysis and a random effects meta-regression model was performed. It adhered to the PRISMA (Preferred Reporting Items for Systematic Reviews and Meta-Analyses) guidelines ${ }^{9}$ and followed the recommendations for ethical publishing of systematic reviews proposed by Wager and Wiffen ${ }^{10}$. Prior to its initiation, the study was registered in the PROSPERO database (CRD42020170412).

Search strategy. Between February and March 2020, two independent investigators (JW, LM) performed a systematic literature search. Articles matching the research question were identified using MEDLINE (PubMed), CochraneCentral, Web of Science and Google Scholar. The terms for all databases were similar but modified according to the requirements of the respective search masks. As an example, the term used for PubMed was: 'high-intensity AND (functional OR body weight) AND (exercise OR training OR workout OR circuit OR conditioning) NOT acute. For Google Scholar, an approach described in previous systematic reviews of our work group $^{11-13}$ was used. Briefly, the first 100 hits displaying the most relevant findings in regards to the entered term were screened for relevant articles in order to complement the results of the other database searches. In addition, the reference lists of all included studies were checked in order to identify further potentially eligible papers ${ }^{14}$.

Inclusion criteria. Randomized controlled trials (crossover or parallel group design) with accessible full text were considered for inclusion. Further criteria were (1) enrolment of healthy individuals, (2) performance of high-intensity functional exercise, (3) testing of chronic effects (minimum of 4 weeks training) on markers of strength, endurance or balance (4) control against inactivity, strength, endurance or balance exercise as well as (5) publication in English language and in a peer-reviewed journal. Interventions were classified as HIFT if they were performed at high relative training intensities, aimed to improve multiple motor functions (e.g. strength, endurance, balance) and included multiple different functional whole-body movements (e.g. jumps, squats, burpees, push-up, running in place). All studies investigating acute effects or other training methods (including combined treatments), lacking the control group types listed in (4) or including persons with diseases were excluded.

Data extraction. Using a standardised assessment sheet, two investigators (JW, LM) independently extracted the following data from included papers: study design, sample size, participant characteristics, interventions including their characteristics (see below), measured outcomes and results (pre-post changes plus standard deviations of each intervention arm). Outcomes of the meta-analysis were strength, endurance and balance. If a study reported more than one strength (e.g., shoulder and leg), endurance (e.g. Vo ${ }^{2}$ max and Bruce test) or balance measure, all respective effect sizes (ES) were extracted.

Data synthesis and statistics. Data from both crossover and parallel-group trials were included. For each intervention arm of parallel-group studies, the mean pre- to post-test changes plus standard deviations (SD) were retrieved. If reporting was incomplete (i.e. missing SDs of the changes from baseline), the required information was requested from the corresponding authors of the trials. If no values could be obtained, missing data were (1) determined from figures or $\mathrm{p}$ values/t values/standard errors or (2) imputed according to the recommendations of the Cochrane handbook, using the formula $\mathrm{SD}_{\text {change }}=\sqrt{ }\left(\mathrm{SD}^{2}\right.$ baseline $+\mathrm{SD}^{2}$ postintervention $)-(2 \times \mathrm{Corr} \times \mathrm{SD}$ baseline $\times \mathrm{SD}$ postintervention), where Corr $=0.7$. The value chosen for Corr represents a conservative estimate of the correlation between the baseline and post-treatment SDs ${ }^{15}$. For crossover trials, the SD of the difference between the two relevant condition's pre-post changes, the correlation of the respective pre-post changes as well as the standard error were computed. If the correlation coefficient for the conditions's pre-post changes could not be extracted from publications or calculated from raw data, a conservative value of 0.5 was assumed, which also fits with the known correlations of the other included studies. When combining the results from parallel group and crossover studies, we used appropriate formulae for standardized mean ES and standard errors ${ }^{16}$.

The following potential moderators of the treatment effect were dichotomized (for details refer to tables): program duration (weeks), session duration (mins), total program volume (mins), rest interval duration (seconds), age (years) and sex (female and male). The choice of the tested moderators was based on three criteria ${ }^{17}$. Firstly, they had to be clearly reported in at least five studies. Secondly, variation had to be present between the levels of a moderator. For instance, if all studies would have stated the sex of the participants, a moderator analysis would have been impossible if only males were included in these studies. Finally, there had to be a plausible theory as to how a moderator would influence the treatment outcome. For instance, it may be assumed that age, with its two moderator levels old and young would lead to a varying treatment effectiveness.

A multilevel meta-analysis with a robust random effects meta-regression model ${ }^{18}$ was used to pool the standardized mean differences (SMD) and 95\% confidence intervals (CI) between HIFT and no-exercise control (NEX), HIFT and moderate continuous aerobic training (MCT), HIFT and high-intensity interval training (HIIT), HIFT and resistance training (RES) as well as HIFT and balance training (BAL). Dependency of ES was 


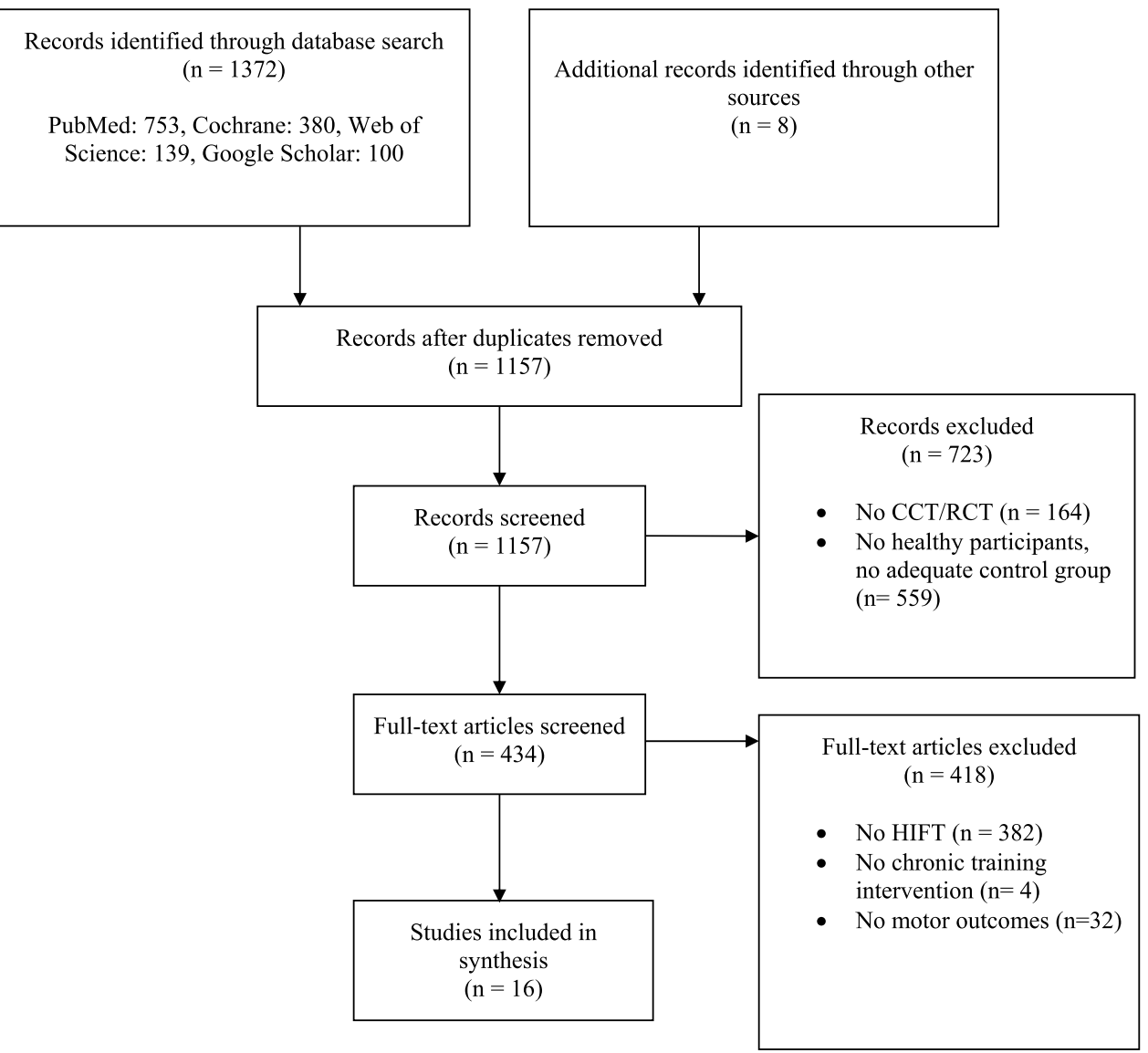

Figure 1. PRISMA chart of the study flow. CCT controlled clinical trial, RCT randomized controlled trial, HIFT high-intensity functional training.

taken into account by nesting the term 'study' as a random factor in the model. Potential moderators were identified with separate models: (1) estimating the significance of each level by means of the $95 \%$ CI and (2) testing for differences between the respective levels ${ }^{13,19}$. The between-study variance component was determined by means of $\mathrm{Tau}^{2}$, using the method-of-moments estimate; for within-study variance (more than one dependent effect size), omega $^{2}\left(\omega^{2}\right)$ was calculated ${ }^{18}$. Pooled effect sizes were interpreted as small $(\mathrm{SMD}=0.2)$, modreate $(\mathrm{SMD}=0.5)$ or large $(\mathrm{SMD}=0.8)^{20}$. P values $<0.05$ were considered significant. The software employed was $\mathrm{R}$ ( $\mathrm{R}$ Foundation for Statistical Computing, Vienna, Austria), packages meta (G Schwarzer) and robumeta (version $2.0^{21}$ ).

Risk of bias and study quality. Publication bias was checked by means of visual inspection of funnel plots (ES against standard errors) and optional sensitivity analyses excluding outliers if at least $10 \mathrm{ES}$ were available $\mathrm{e}^{22}$. The methodological quality of the included studies was assessed by means of the PEDro scale, which has been shown to represent a high reliability and validity for this purpose ${ }^{23-25}$. The sum score of the instrument is calculated from 10 items capturing potential sources of bias. Two independent investigators (JW, LM) performed the quality scoring.

\section{Results}

Search results. A flow diagram of the literature search is displayed in Fig. 1. The algorithms used returned a total of 1372 records. Sixteen studies ${ }^{5,26-40}$ (Table 1) met the eligibility criteria and were included in the review.

Characteristics of the studies. The 16 papers collectively evaluated 864 participants (302 men and 458 women, distribution unknown for two studies) with mean ages ranging from 11.7 to 72.8 years (Table 1). Most trials $(n=15)$ used a parallel-group design while only one employed a crossover design.

Ten papers ${ }^{26-31,35,38-40}$ compared HIFT vs. NEX, four papers ${ }^{32,33,36,37}$ examined HIFT vs. HIIT, seven papers investigated HIFT vs. MCT ${ }^{5,31-34,39,40}$. No studies comparing HIFT vs. RES or BAL were found.

Complete data were available, obtained or extracted from figures for nine studies ${ }^{5,31-33,36-40}$, whilst imputation of standard deviations was needed for 7 trials $^{26-30,34,35}$.

Methodological quality. The two reviewers agreed in 155 (96.9\%) of the 160 criteria scored by means of the PEDro scale. All disagreements were resolved by discussion. The methodological quality of the 16 included 


\begin{tabular}{|c|c|c|c|c|c|}
\hline Study & Design & Participants & HIFT protocol/control & Duration & Outcomes \\
\hline Engel et al. ${ }^{26}$ & $\begin{array}{l}\text { Parallel-group } \\
\text { 1: } \operatorname{HIFT}(\mathrm{n}=10) \\
\text { 2: } \operatorname{NEX~}(\mathrm{n}=10)\end{array}$ & $\begin{array}{l}\mathrm{n}=20 \text { healthy, moder- } \\
\text { ately trained adults }(10 \\
\text { females and } 10 \text { males; age } \\
36.2 \pm 11.1 \text { years; BMI: } \\
\left.23.9 \pm 3.7 \mathrm{~kg} / \mathrm{m}^{2}\right)\end{array}$ & $\begin{array}{l}\text { 1: } 2 \mathrm{x} / \text { week, } 30 \text { min, eight } \\
\text { whole-body exercises with } \\
\text { suspension trainer (e.g. } \\
\text { squats, burpees, jumping } \\
\text { jacks, chest press, mountain } \\
\text { climbers), } 20 \mathrm{~s} \text { all-out exer- } \\
\text { cise, } 10 \mathrm{~s} \text { rest } \\
2:-\end{array}$ & 8 weeks & $\begin{array}{l}\text { Strength: } \mathrm{LP}, \mathrm{CP}, \mathrm{PD}, \mathrm{BE} \\
\text { Endurance: } \mathrm{V}_{\max }\end{array}$ \\
\hline Engel et al. ${ }^{27}$ & $\begin{array}{l}\text { Parallel-Group } \\
\text { 1: } \operatorname{HIFT}(\mathrm{n}=17) \\
\text { 2: } \operatorname{NEX~}(\mathrm{n}=18)\end{array}$ & $\begin{array}{l}\mathrm{n}=35 \text { secondary school chil- } \\
\text { dren }(24 \text { males, } 11 \text { females, } \\
11.7 \pm 0.3 \text { years })\end{array}$ & $\begin{array}{l}\text { 1: } 4 \mathrm{x} / \text { week, } 6 \text { min, circuit- } \\
\text { like all-out whole-body } \\
\text { exercise (e.g. planks, burpees, } \\
\text { skippings), varying durations } \\
\text { and breaks } \\
\text { 2:- }\end{array}$ & 4 weeks & $\begin{array}{l}\text { Strength and endurance: } \\
\text { push-ups, sit-ups, standing } \\
\text { LJ, Lateral jumps, 20-m } \\
\text { sprint, Balance: steps } \\
\text { backwards } \\
\text { Endurance: 6-min run }\end{array}$ \\
\hline Schmidt et al. ${ }^{28}$ & $\begin{array}{l}\text { Parallel-Group } \\
\text { 1: HIFT-7 }(\mathrm{n}=32) \\
\text { 2: HIFT-14 }(\mathrm{n}=28) \\
\text { 3: } \operatorname{NEX~}(\mathrm{n}=36)\end{array}$ & $\begin{array}{l}\mathrm{n}=96 \text {, active collage students, } \\
(53 \text { females, } 43 \text { males, } \\
\text { age: } 18-24 \text { years }) \\
1: 17 \text { females, } 15 \text { males } \\
\text { 2: } 15 \text { females, } 13 \text { males } \\
3: 21 \text { females, } 15 \text { males }\end{array}$ & $\begin{array}{l}\text { 1: } 3 \mathrm{x} / \text { week, } 7 \text { min, whole- } \\
\text { body exercises (e.g. jumping } \\
\text { jacks, wall sit, push-ups, } \\
\text { abdominal crunch, step-up } \\
\text { chair, squat), } 30 \text { s exercise, } \\
10 \text { rest } \\
\text { 2: Identical to group } 1 \text { but } \\
\text { with } 14 \text { min duration in } \\
\text { weeks } 5-8 \\
3:-\end{array}$ & 8 weeks & $\begin{array}{l}\text { Strength and endurance: } \\
\text { HST, push-ups } \\
\text { Endurance: } \mathrm{VO}_{2} \max \end{array}$ \\
\hline Ballesta-Garcia et al. ${ }^{29}$ & $\begin{array}{l}\text { Parallel-Group } \\
1: \operatorname{HIFT}(\mathrm{n}=18) \\
2: \operatorname{NEX}(\mathrm{n}=18)\end{array}$ & $\begin{array}{l}\mathrm{n}=36 \text { females } \\
(67.8 \pm 6.2 \text { years }) \\
\text { 1: Age: } 66.3 \pm 5.4 \text { years } \\
\text { 2: Age: } 67.4 \pm 5.7 \text { years }\end{array}$ & $\begin{array}{l}\text { 1: } 2 x / \text { week, variable dura- } \\
\text { tion/intensity, whole-body } \\
\text { exercises (e.g. jumping jacks, } \\
\text { squats, walking) } \\
\text { 2:- }\end{array}$ & 18 weeks & $\begin{array}{l}\text { Strength: arm curl test, STS- } \\
\text { 30, HST } \\
\text { Balance: 1LST, TUG } \\
\text { Endurance: HR, 6MWT }\end{array}$ \\
\hline Sperlich et al. ${ }^{30}$ & $\begin{array}{l}\text { Crossover (wait-control } \\
\text { intervention }= \\
\text { 1: NEX /HIFT1 }(\mathrm{n}=12) \\
\text { 2: NEX/HIFT2 }(\mathrm{n}=12)\end{array}$ & $\begin{array}{l}\mathrm{n}=24 \text { untrained adults } \\
\text { (14 females, } 10 \text { males; } \\
25 \pm 5 \text { years) } \\
\text { 1: } 7 \text { females, } 5 \text { males } \\
\text { 2: } 7 \text { females, } 5 \text { males }\end{array}$ & $\begin{array}{l}\text { 1: } 1 \mathrm{x} / \text { day, } 6 \text { min, whole-body } \\
\text { exercise (e.g. burpees, leg } \\
\text { levers, push-ups) } \\
\text { 2: Identical to group } 1 \text { but } \\
\text { 2x/day }\end{array}$ & $\begin{array}{l}8 \text { weeks }(4 \text { weeks } \\
\text { NEX/4 weeks HIFT) } \\
1\end{array}$ & $\begin{array}{l}\text { Strength: push-ups, leg } \\
\text { levers, burpees, one-legged } \\
\text { squat, skipping } \\
\text { Endurance: } \mathrm{Vo}_{2} \max \end{array}$ \\
\hline McRae et al. ${ }^{31}$ & $\begin{array}{l}\text { Parallel-Group } \\
\text { 1: MCT }(\mathrm{n}=7) \\
\text { 2: } \operatorname{HIFT}(\mathrm{n}=7) \\
\text { 3: } \operatorname{NEX}(\mathrm{n}=8)\end{array}$ & $\begin{array}{l}\mathrm{n}=22 \text { recreationally active } \\
\text { females } \\
1: 21.1 \pm 2.8 \text { years } \\
2: 20.7 \pm 1 \text { years } \\
3: 19.2 \pm 0.9 \text { years }\end{array}$ & $\begin{array}{l}\text { 1: } 4 \mathrm{x} / \text { week, } 30 \mathrm{~min} \text {, treadmill } \\
\text { running at } 85 \% \mathrm{HR}_{\max } \\
\text { 2: } 4 \mathrm{x} / \text { week, } 8 \times 20 \mathrm{~s} \text { single } \\
\text { exercise }(\mathrm{e} . \mathrm{g} . \text { burpees, } \\
\text { jumping jacks, mountain } \\
\text { climbers) } \\
\text { 3:- }\end{array}$ & 4 weeks & $\begin{array}{l}\text { Strength: LE, LC, lateral PD, } \\
\text { CP, push-ups, sit-ups, BE } \\
\text { Endurance: } \mathrm{Vo}_{2} \max , \text { Bruce } \\
\text { protocol }\end{array}$ \\
\hline Schaun et al. ${ }^{32}$ & $\begin{array}{l}\text { Parallel-Group } \\
\text { 1: } \operatorname{HIIT}(\mathrm{n}=15) \\
\text { 2: HIFT }(\mathrm{n}=12) \\
\text { 3: } \operatorname{MCT}(\mathrm{n}=14)\end{array}$ & $\begin{array}{l}\mathrm{n}=41 \text { recreationally active } \\
\text { males }(23.7 \pm 0.7 \text { years })\end{array}$ & $\begin{array}{l}1: 3 \mathrm{x} / \text { week, } 8 \times 20 \mathrm{~s} \text { treadmill } \\
\text { running at } 130 \% \text { of the } \\
\text { velocity associated to } \\
\mathrm{VO}_{2} \text { max, } 10 \mathrm{~s} \text { rest } \\
2: 3 \mathrm{x} / \text { week, } 8 \times 20 \mathrm{~s}, 4 \text { calis- } \\
\text { thenics exercises (burpees, } \\
\text { mountain climbers, squat, } \\
\text { thrusts), } 10 \mathrm{~s} \text { rest } \\
3: 3 \mathrm{x} / \text { week, } 30 \mathrm{~min} \text {, treadmill } \\
\text { running at } 90-95 \% \text { of the HR } \\
\text { associated to the ventilatory } \\
\text { threshold }\end{array}$ & 16 weeks & $\begin{array}{l}\text { Strength: CMJ, SJ, EMG } \\
\text { signals of RF \& VL }\end{array}$ \\
\hline Schaun et al. ${ }^{33}$ & $\begin{array}{l}\text { Parallel-Group } \\
\text { 1: } \operatorname{HIIT}(\mathrm{n}=15) \\
\text { 2: HIFT }(\mathrm{n}=12) \\
\text { 3: } \operatorname{MCT}(\mathrm{n}=14)\end{array}$ & $\begin{array}{l}\mathrm{n}=41 \text { recreationally active } \\
\text { males }(23.7 \pm 0.7 \text { years })\end{array}$ & $\begin{array}{l}1: 3 \mathrm{x} / \text { week, } 8 \times 20 \mathrm{~s} \text { treadmill } \\
\text { running at } 130 \% \text { of the } \\
\text { velocity associated to } \\
\mathrm{VO}_{2} \text { max, } 10 \mathrm{~s} \text { rest } \\
2: 3 \mathrm{x} / \text { week, } 8 \times 20 \mathrm{~s}, 4 \text { calis- } \\
\text { thenics exercises (burpees, } \\
\text { mountain climbers, squat, } \\
\text { thrusts), } 10 \mathrm{~s} \text { rest } \\
3: 3 \mathrm{x} / \text { week, } 30 \mathrm{~min} \text {, treadmill } \\
\text { running at } 90-95 \% \text { of the } \\
\mathrm{HR} \text { associated to the second } \\
\text { ventilatory threshold }\end{array}$ & 16 weeks & Endurance: $\mathrm{Vo}_{2} \max$ \\
\hline Evangelista et al. ${ }^{34}$ & $\begin{array}{l}\text { Parallel-Group } \\
\text { 1: HIFT }(\mathrm{n}=14) \\
2: \operatorname{MCT}(\mathrm{n}=11)\end{array}$ & $\begin{array}{l}\mathrm{n}=25 \text { physically active } \\
\text { participants (unknown sex } \\
\text { and age) }\end{array}$ & $\begin{array}{l}\text { 1: } 3 \mathrm{x} / \text { week, } 20 \text { sets of } 30 \mathrm{~s} \text { all- } \\
\text { out exercise, } 30 \mathrm{~s} \text { rest (jump- } \\
\text { ing jacks, mountain climbers, } \\
\text { burpees, squat jumps) } \\
2: 3 \mathrm{x} / \text { week, } 25 \text { min running } \\
\left(80 \% \mathrm{HR}_{\max }\right)\end{array}$ & 6 weeks & Strength: sit-ups, push-ups \\
\hline Garcia-Pinillos et al. ${ }^{35}$ & $\begin{array}{l}\text { Parallel-Group } \\
\text { 1: HIFT }(n=47) \\
2: \operatorname{NEX~}(n=43)\end{array}$ & $\begin{array}{l}\mathrm{n}=90 \text { active adults } \\
(64 \text { females, } 26 \text { males; } \\
72.8 \pm 5.7 \text { years })\end{array}$ & $\begin{array}{l}\text { 1: } 3 \mathrm{x} / \text { week, } 35-40 \mathrm{~min} \text {, } \\
\text { high-intensity circuit } \\
\text { strength training combined } \\
\text { with high-intensity interval } \\
\text { endurance training as active } \\
\text { recovery (e.g. medicine ball } \\
\text { throws, farmer's walk, sit to } \\
\text { stand) } \\
\text { 2:- }\end{array}$ & 12 weeks & $\begin{array}{l}\text { Strength: HST, STS-30 } \\
\text { Balance: CoP }\end{array}$ \\
\hline
\end{tabular}




\begin{tabular}{|c|c|c|c|c|c|}
\hline Study & Design & Participants & HIFT protocol/control & Duration & Outcomes \\
\hline Menz et al. ${ }^{36}$ & $\begin{array}{l}\text { Parallel-Group } \\
\text { 1: HIIT }(\mathrm{n}=8) \\
\text { 2: } \operatorname{HIFT}(\mathrm{n}=7)\end{array}$ & $\begin{array}{l}\mathrm{n}=15 \text { moderately trained } \\
\text { adults }(25.6 \pm 2.6 \text { years }) \\
1: 6 \text { females, } 2 \text { males } \\
2: 5 \text { females, } 2 \text { males }\end{array}$ & $\begin{array}{l}3-4 \text { sets; } 8 \times 20 \mathrm{~s} \text { all-out } \\
\text { exercise, } 10 \mathrm{~s} \text { rest } \\
\text { 1: running HIIT } \\
\text { 2: functional HIIT with body } \\
\text { weight }\end{array}$ & 4 weeks & $\begin{array}{l}\text { Strength: push-ups, toes to } \\
\text { bar, BJ, burpees } \\
\text { Endurance: } \mathrm{Vo}_{2} \max , \mathrm{HR}_{\max } \\
\mathrm{BLA}_{\max }\end{array}$ \\
\hline Buckley et al. ${ }^{37}$ & $\begin{array}{l}\text { Parallel-Group } \\
1: \text { HITT }(\mathrm{n}=14) \\
\text { 2: HIFT }(\mathrm{n}=14)\end{array}$ & $\begin{array}{l}\mathrm{n}=28 \text { recreationally active } \\
\text { females }(24.7 \pm 5.4 \text { years }) \\
1: 24.3 \pm 5.2 \text { years } \\
2: 25.1 \pm 5.6 \text { years }\end{array}$ & $\begin{array}{l}1: 3 \mathrm{x} / \text { week, } 6 \text { sets of } 60 \mathrm{~s} \text { all } \\
\text { out intensity rowing, } 3 \mathrm{~min} \\
\text { rest } \\
2: 3 \mathrm{x} / \text { week, } 6 \text { sets of } 60 \mathrm{~s} \\
\text { all out intensity workout } \\
\text { (strength exercise }(4-6 \text { repe- } \\
\text { titions), accessory movement } \\
\text { (8-10 repetitions), metabolic } \\
\text { component), } 3 \text { min rest }\end{array}$ & 6 weeks & $\begin{array}{l}\text { Strength: squat, } \mathrm{CP}, \mathrm{DL}, \mathrm{BJ} \\
\text { Endurance: } \mathrm{Vo}_{2} \mathrm{max}, \\
\text { anaerobic power, anaerobic } \\
\text { capacity, squat endurance }\end{array}$ \\
\hline Greenlee et al. ${ }^{38}$ & $\begin{array}{l}\text { Parallel-Group } \\
\text { 1: HIFT }(\mathrm{n}=129) \\
\text { 2: } \operatorname{NEX}(\mathrm{n}=129)\end{array}$ & $\begin{array}{l}\mathrm{n}=258 \text { adults } \\
1: 61 \text { females, } 66 \text { males; } \\
24.7 \pm 5.6 \text { years } \\
2: 63 \text { females, } 66 \text { males; } \\
24.3 \pm 5.7 \text { years }\end{array}$ & $\begin{array}{l}\text { 1: varying frequency and } \\
\text { volume, e.g. resistance band } \\
\text { exercixes, rope skipping, } \\
\text { high-intensity cardiorespira- } \\
\text { tory exercises, } \\
\text { 2:- } \\
\end{array}$ & 16 weeks & $\begin{array}{l}\text { Strength: } \\
\text { Push-ups, towers, } \mathrm{LJ} \\
\text { Endurance: } \mathrm{Vo}_{2} \max , 1.5 \text {-mile } \\
\text { run }\end{array}$ \\
\hline Jimenez-Garcia et al. $^{39}$ & $\begin{array}{l}\text { Parallel-Group } \\
\text { 1: HIFT }(\mathrm{n}=26) \\
\text { 2: MCT }(\mathrm{n}=24) \\
\text { 3: } \operatorname{NEX~}(\mathrm{n}=23)\end{array}$ & $\begin{array}{l}\mathrm{n}=73 \text { recreationally active } \\
\text { adults (unknown sex) } \\
1: 68.2 \pm 3.0 \text { years } \\
2: 68.8 \pm 6.0 \text { years } \\
3: 68.5 \pm 6.3 \text { years }\end{array}$ & $\begin{array}{l}\text { 1:2x/week, } 4 \text { intervalls of } \\
4 \text { min suspension training } \\
\text { exercises at } 90-95 \% \mathrm{HR}_{\max }, \\
3 \text { min active rest } \\
\text { 2: same as in group one but } \\
\text { with } 50-70 \% \mathrm{HR}_{\max } \\
\text { 3: guidelines to encourage } \\
\text { phyical activity }\end{array}$ & 12 weeks & Strength: HST, TUG \\
\hline Wilke et al. ${ }^{5}$ & $\begin{array}{l}\text { Parallel-Group } \\
\text { 1: HIFT }(\mathrm{n}=20) \\
\text { 2: } \text { MCT }(\mathrm{n}=13)\end{array}$ & $\begin{array}{l}\mathrm{n}=33 \text { untrained adults } \\
1: 11 \text { females, } 9 \text { males; } \\
24.5 \pm 6.3 \text { years } \\
2: 10 \text { females, } 3 \text { males; } \\
23.7 \pm 3.2 \text { years }\end{array}$ & $\begin{array}{l}\text { 1: 3x/week, } 15 \text { min, whole- } \\
\text { body exercises (e.g. squats, } \\
\text { burpees, push-up), } 20 \mathrm{~s} \text { all- } \\
\text { out exercise, } 10 \mathrm{~s} \text { rest } \\
\text { 2: } 3 \mathrm{x} / \text { week, } 50 \mathrm{~min} \text {, walking } \\
\text { at } 50-60 \% \text { HRR }\end{array}$ & 6 weeks & $\begin{array}{l}\text { Strength: } \\
\text { SLHD, CMJ, RSI, LP, CP Bal- } \\
\text { ance: CoP } \\
\text { Endurance: } \mathrm{Vo}_{2} \max \end{array}$ \\
\hline Islam et al. ${ }^{40}$ & $\begin{array}{l}\text { Parallel-Group } \\
\text { 1: HIFT }(\mathrm{n}=26) \\
\text { 2: MCT }(\mathrm{n}=27) \\
\text { 3: } \operatorname{NEX}(\mathrm{n}=15)\end{array}$ & $\begin{array}{l}\mathrm{n}=68 \text { inactive adults, } \\
51 \text { females, } 17 \text { males, } \\
21 \pm 3 \text { years }\end{array}$ & $\begin{array}{l}\text { 1: } 4 \mathrm{x} / \text { week, } 4 \times 20 \mathrm{~s} \text { whole- } \\
\text { body exercises (burpees, } \\
\text { mountain climber, jumping } \\
\text { jacks, sqaut) performed at } \\
20 \mathrm{~s} \text { intervals with } 10 \mathrm{~s} \text { rest } \\
\text { 2: } 4 \mathrm{x} / \text { week, } 30 \mathrm{~min} \text {, running } \\
\text { on treadmill at } 85 \% \mathrm{HR}_{\max } \\
\text { 3:- }\end{array}$ & 4 weeks & $\begin{array}{l}\text { Endurance: } \mathrm{VO}_{2} \max , 5 \mathrm{~km} \\
\text { TT, TTF }\end{array}$ \\
\hline
\end{tabular}

Table 1. Characteristics of the included studies. Age data are means \pm standard deviations. HIFT highintensity functional training, $M C T$ moderate continuous training, NEX no exercise, min minutes, $s$ seconds, $L P$ leg press, $C P$ chest press, $P D$ pull downs, $B E$ back extensions, $V_{\max }$ maximal running speed, $H R R$ heart rate reserve, $H R$ heart rate, $L J$ long jump, $H S T$ hand strength, $V_{2}$ max maximal rate of oxygen consumption, STS3030 s. sit-to-stand, TUG timed up and go, $1 L S T$ one leg stance, $6 M W T 6$ min walking test, LE leg extensions, $L C$ leg curls, $C M J$ counter movement jump, SJ squat jump, EMG electromyography, $R F$ rectus femoris, $V L$ vastus lateralis, $C o P$ center of pressure, $B J$ broad jump, $B L A_{\max }$ maximal blood lactate concentration, $D L$ dead lift, SLHD single leg hop distance, $R S I$ reactive strength index, $T T$ time trial, $T T F$ time to fatique.

papers ranged from 4 to 7 out of 10 and their mean scores, in sum (5.9 \pm 0.9$)$, were classified as moderate. Most studies reported randomization, between-group comparisons and point estimates plus variability indicators, had comparable baseline values and used intention-to-treat. In contrast, only a few studies used concealed allocation as well as therapist/instructor, participant or assessor blinding (Table 2).

HIFT vs. no exercise. For endurance capacity, a small ES favouring HIFT over NEX was found (SMD: 0.42, 95\% CI: 0.07 to $0.78, p=0.03$, Tau ${ }^{2}: 0.19, \omega^{2}: 0, n=8$ studies, $n=18$ ES, Fig. 2). Similarly, a moderate-magnitude ES suggested superiority of HIFT regarding strength (SMD: 0.60, 95\% CI 0.02-1.18, p=0.04, Tau ${ }^{2}: 0.50, \omega^{2}: 0$, $\mathrm{n}=7$ studies, $\mathrm{n}=44 \mathrm{ES}$, Fig. 3 ). In contrast to the above outcomes, only very few studies assessed balance. The pooled comparison did not yield a significant difference between HIFT and NEX (SMD: - 0.10, 95\% CI - 1.13 to $0.92, \mathrm{p}=0.42, \mathrm{Tau}^{2}: 0, \omega^{2}: 0.01, \mathrm{n}=2$ studies, $\mathrm{n}=3 \mathrm{ES}$, Fig. 4 ).

In most cases (Table 3), no differences between the levels of the tested moderator variables were found ( $p>0.05$, Table 3$)$. The only exeception was sex: Females appeared to respond more strongly to HIFT with regard to endurance capacity when compared to mixed samples $(\mathrm{p}=0.02)$. A comparison against men only was impossible due to a lack of data. The same applied for the moderator analysis of HIFT vs. NEX regarding balance.

HIFT vs. endurance training. Meta-analytic pooling did not reveal any differences between HIFT and MCT with regard to endurance capacity (SMD: $-0.11,95 \% \mathrm{CI}-1.17$ to $0.95, \mathrm{p}=0.75$, Tau ${ }^{2}: 0.29, \omega^{2}: 0.06, \mathrm{n}=4$ studies, $\mathrm{n}=9$ ES, Fig. 5). Similarly, no differences were found for HIFT vs. HIIT (SMD: $-0.15,95 \%$ CI -1.4 to $1.1, \mathrm{p}=0.66$, Tau ${ }^{2}: 0.048, \omega^{2}: 0, \mathrm{n}=3$ studies, $\mathrm{n}=5 \mathrm{ES}$, Fig. 6). Due to the small number of studies, data were insufficient for moderator analyses. 


\begin{tabular}{|c|c|c|c|c|c|c|c|c|c|c|c|c|}
\hline Study & $\begin{array}{l}\text { Inclusion } \\
\text { criteria }^{\mathrm{a}}\end{array}$ & $\begin{array}{l}\text { Random } \\
\text { allocation }\end{array}$ & $\begin{array}{l}\text { Concealed } \\
\text { allocation }\end{array}$ & $\begin{array}{l}\text { Similarity } \\
\text { at baseline }\end{array}$ & $\begin{array}{l}\text { Subject } \\
\text { blinding }\end{array}$ & $\begin{array}{l}\text { Therapist } \\
\text { blinding }\end{array}$ & $\begin{array}{l}\text { Assessor } \\
\text { blinding }\end{array}$ & $\begin{array}{l}>85 \% \\
\text { follow-up }\end{array}$ & $\begin{array}{l}\text { Intention } \\
\text { to treat } \\
\text { analysis }\end{array}$ & $\begin{array}{l}\text { Between- } \\
\text { group } \\
\text { comparisons }\end{array}$ & $\begin{array}{l}\text { Point } \\
\text { estimates } \\
\text { and } \\
\text { variability }\end{array}$ & $\begin{array}{l}\text { Total } \\
\text { (points) }\end{array}$ \\
\hline Engel et al. ${ }^{26}$ & + & + & - & + & - & - & - & + & + & + & + & 6 \\
\hline Engel et al. ${ }^{27}$ & + & + & - & + & - & - & - & + & + & + & + & 6 \\
\hline $\begin{array}{l}\text { Schmidt } \\
\text { et al. } .^{28}\end{array}$ & + & + & - & + & - & - & - & + & + & + & + & 6 \\
\hline $\begin{array}{l}\text { Ballesta- } \\
\text { Garcia } \\
\text { et al. } .^{29}\end{array}$ & + & + & - & + & - & - & + & - & + & + & + & 6 \\
\hline $\begin{array}{l}\text { Sperlich } \\
\text { et al. }{ }^{30}\end{array}$ & + & + & - & + & - & - & - & + & + & + & + & 6 \\
\hline $\begin{array}{l}\text { McRae } \\
\text { et al. }{ }^{31}\end{array}$ & - & - & - & + & - & - & - & + & - & + & + & 4 \\
\hline $\begin{array}{l}\text { Schaun } \\
\text { et al. }{ }^{32}\end{array}$ & + & + & - & + & - & - & + & + & + & + & + & 7 \\
\hline $\begin{array}{l}\text { Schaun } \\
\text { et al. }\end{array}$ & + & + & - & + & - & - & + & - & + & + & + & 6 \\
\hline $\begin{array}{l}\text { Evangelista } \\
\text { et al. } .^{34}\end{array}$ & + & + & - & + & - & - & - & + & + & + & + & 6 \\
\hline $\begin{array}{l}\text { Garcia-Pin- } \\
\text { illos et al. } .^{35}\end{array}$ & + & + & - & + & - & - & - & + & + & + & + & 6 \\
\hline Menz et al. ${ }^{36}$ & + & + & - & + & - & - & - & - & + & + & + & 5 \\
\hline $\begin{array}{l}\text { Buckley } \\
\text { et al. } .^{37}\end{array}$ & - & + & - & + & - & + & - & + & + & + & + & 7 \\
\hline $\begin{array}{l}\text { Greenlee } \\
\text { et al. }{ }^{38}\end{array}$ & + & + & - & + & - & - & - & - & - & + & + & 4 \\
\hline $\begin{array}{l}\text { Jimenez- } \\
\text { Garcia } \\
\text { et al. }{ }^{39}\end{array}$ & + & + & + & + & - & - & - & + & + & + & + & 7 \\
\hline Wilke et al. ${ }^{5}$ & + & + & - & + & - & - & - & + & + & + & + & 6 \\
\hline Islam et al. ${ }^{40}$ & + & + & - & + & - & - & - & + & + & + & + & 6 \\
\hline
\end{tabular}

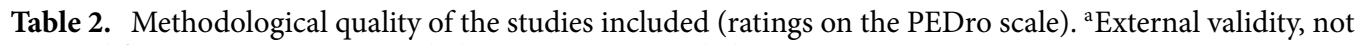
counted for score, $+=$ point awarded, $-=$ no point awarded.

Risk of bias. Visual inspection of the funnel plots (Fig. 7 for an example) suggested a potential reporting bias for HIFT vs. NEX regarding both, endurance and muscle strength markers due to a large standard error and small sample size in few studies. Sensitivity analyses without these trials, however, did not modify the general conclusions. Due to small numbers of ES $(<10)$, reporting bias could not be assessed for HIFT vs. MCT and HIFT vs. HIIT.

\section{Discussion}

This systematic review with meta-analysis presents the first differentiated summary of the long-term effects of HIFT on markers of motor performance. Our results demonstrate that related interventions, performed over multiple weeks, induce small to moderate improvements in endurance and strength capacities. Regarding the former, HIFT is neither superior nor inferior to traditional exercise regimes such as aerobic training or HIIT.

The mechanisms by which HIFT acts on the body are a matter of debate. The performance of repeated wholebody exercises provokes a cardioplumonary output which is comparable to traditional endurance training: Tibana et al. ${ }^{41,42}$ found maximum heart rates of more than 180 beats per minute and blood lactate concentrations of 11-18 mmol/l following two HIFT workouts. It is therefore plausible that repeated engagement in HIFT improves endurance capacity. In resistance training, time under tension represents an essential parameter steering protein synthesis ${ }^{43}$. One hallmark of HIFT is the execution of high repetition numbers with short breaks ${ }^{2}$. As time under tension is rather long, this may create a potent stimulus. High metabolic stress represents another important trigger for muscle hypertrophy. After acute bouts of HIFT, elevated pro-inflammatory cytokines (IL6) were observed ${ }^{41}$. This, in concert with the high blood lactate levels, may lead to metabolic conditions which are favourable for muscle adaptations.

The findings of our meta-analysis have significant implications for health professionals and fitness coaches. Firstly, based on the available evidence, HIFT appears to represent a viable alternative to conventional training methods if clients and patients do not like traditional resistance or endurance training. Secondly, besides general aversion towards sport or specific exercise regimes, many inactive individuals state lack of time as a main brarrier to engage in physical activity ${ }^{3,4}$. As HIFT can be performed at short overall durations and, contrary to HIIT, seems to address multiple motor abilities, it represents an intriguing option when aiming to increase participation in and adherence to physical acivity programs. This particularly applies because formerly inactive 
Ballesta-Garcia et al. [28]

6-minute walking test

$\begin{array}{ll}-1.04 & 0.40\end{array}$

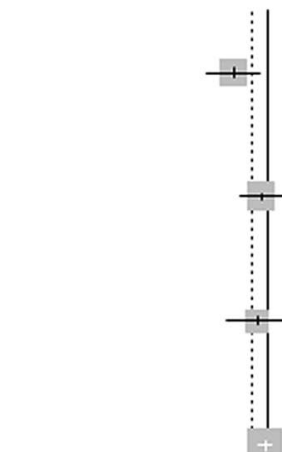

Engel et al.

6-minute run [26]

$-0.16 \quad 0.35$

Engel et al. [25]

Running velocity

$\begin{array}{ll}-0.28 & 0.48\end{array}$

Greenlee et al. [37]

$\mathrm{VO}_{2} \max$

$\begin{array}{ll}-0.06 & 0.13\end{array}$

Islam et al. [40]

$\mathrm{VO}_{2} \max$

$\begin{array}{ll}-0.18 & 0.33\end{array}$

$5 \mathrm{~km}$ time trial

$\begin{array}{ll}-0.58 & 0.34\end{array}$

Time to fatigue

$-0.84 \quad 0.35$

McRae et al. [30]

Bruce test

$\begin{array}{ll}-1.33 & 0.64\end{array}$

$\mathrm{VO}_{2} \max$

$\begin{array}{ll}-5.01 & 1.28\end{array}$

Cunningham test

$\begin{array}{ll}-1.13 & 0.62\end{array}$

Schmidt et al. [27]

$\mathrm{VO}_{2} \max \delta 7 \mathrm{~min}$

$0.22 \quad 0.38$

$\mathrm{VO}_{2} \mathrm{max}$ of $14 \mathrm{~min}$

$\begin{array}{ll}-0.30 & 0.39\end{array}$

$\mathrm{VO}_{2} \mathrm{max} 97 \mathrm{~min}$

$\begin{array}{ll}-0.65 & 0.34\end{array}$

$\mathrm{VO}_{2} \max 914 \mathrm{~min}$

$\begin{array}{ll}-0.96 & 0.37\end{array}$

Sperlich et al. [29]

$\mathrm{VO}_{2} \max 1 \mathrm{x}$

$\begin{array}{ll}0.16 & 0.22\end{array}$

$\mathrm{VO}_{2} \max 2 \mathrm{x}$

$\begin{array}{lll}0.15 & 0.22\end{array}$

Skippings $1 \mathrm{x}$

$\begin{array}{ll}-0.81 & 0.25\end{array}$

Skippings $2 \mathrm{x}$

$0.00 \quad 0.21$

Pooled (RE)

$-0.42$

$[-1.82 ;-0.25]$

$[-0.84 ; 0.53]$

$[-1.22 ; 0.65]$

$[-0.30 ; 0.19]$

$[-0.83 ; 0.47]$

$[-1.24 ; 0.09]$

$[-1.52 ;-0.16]$

$[-2.58 ;-0.07]$

$[-7.52 ;-2.50]$

$[-2.35 ; 0.08]$

$[-0.52 ; 0.97]$

$[-1.07 ; 0.48]$

$[-1.32 ; 0.03]$

$[-1.69 ;-0.24]$

$[-0.26 ; 0.58]$

$[-0.28 ; 0.57]$

$[-1.30 ;-0.31]$

$[-0.41 ; 0.42]$

$[-0.07 ;-0.78]$

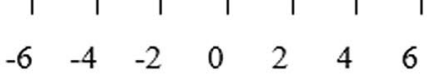

Favours HIFT

Favours NEX

Figure 2. Effects of high-intensity-functional training (HIFT) vs. no exercise (NEX) on markers of endurance performance. Forest plots with pooled standardized mean differences (SMD), standard errors (SE) and 95\% confidence intervals (CI) are displayed. $R E$ random effects.

persons reported higher levels of exercise enjoyment following HIFT when compared to the often prescribed conventional endurance training ${ }^{5}$.

Despite the promising fields of application, several aspects call for further research. In our review, we focused on markers of strength, endurance and balance, which are of interest for most members of the normal population. However, athletes, in addition, frequently require capacities such as speed or power and hence, it would be intriguing to examine the potential effect of HIFT in this context too. Also, while various studies compared HIFT to NEX as well as HIFT to different types of endurance exercise, there is a lack of trials regarding the comparison against resistance training and balance training. Upcoming studies should hence aim to incorporate these two motor capacitities. Another issue relates to methodological considerations. Inspection of the funnel plots suggested the possibility of a publication bias and hence the true effect sizes may be slightly different to the ones reported here. Although this should be underlined when interpreting the results, for several reasons, funnel plot asymmetry must not be overestimated. In general, it has been shown that a non-normal shape of 


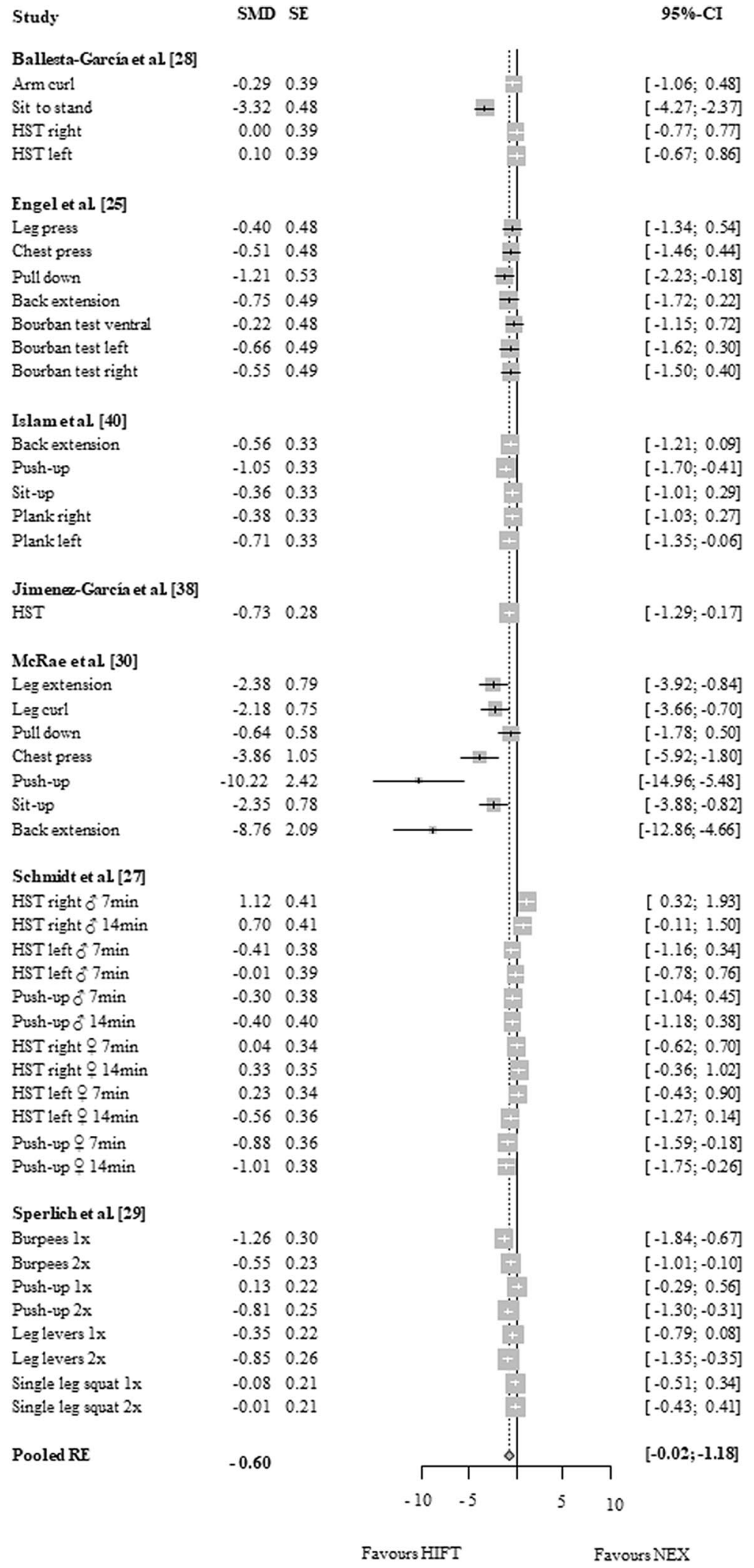

Figure 3. Effects of high-intensity-functional training (HIFT) vs. no exercise (NEX) on markers of muscle strength. Forest plots with pooled standardized mean differences (SMD), standard errors (SE) and 95\% confidence intervals (CI) are displayed. HST hand strength, min minutes, RE random effects. 
Ballesta-García et al. [28]

Single leg stance right

$\begin{array}{ll}0.46 & 0.40\end{array}$

Single leg stance left

$\begin{array}{ll}-0.11 & 0.39\end{array}$

Engel et al. [26]

Steps backwards

$0.00 \quad 0.35$

Pooled RE
0.10

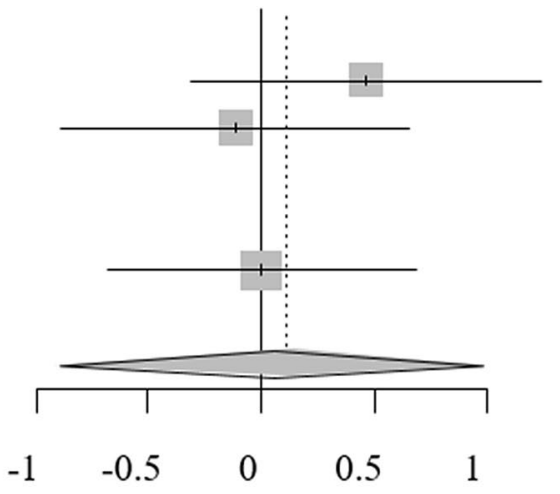

$[-0.32 ; 1.24]$

$[-0.88 ; 0.65]$

$[-0.68 ; 0.68]$

$[-0.92 ; 1.13]$
Favours HIFT
Favours NEX

Figure 4. Effects of high-intensity-functional training (HIFT) vs. no exercise (NEX) on markers of balance. Forest plots with pooled standardized mean differences (SMD), standard errors (SE) and 95\% confidence intervals $(\mathrm{CI})$ are displayed. $R E$ random effects.

the graph represents a hint but not necessarily a proof of presence of bias as it can also be the result of betweenstudy heterogeneity, small-sample study quality, chance and the use of standardized mean differences ${ }^{44,45}$. As our sub-analyses excluding outliers did not modify the main result of the analysis, we conclude that no substantial reporting bias is present. Regarding effect modifiers, with our moderator analysis, we made a strong effort to test the relevance of a plethora of variables. Yet, some others remain to be examined. Only four of the identified studies included older participants and only one was performed in children. It would be reasonable to assume that the treatment response substantially differs as a function of age and hence there is a need to design future trials for children and elderly persons. In a similar way, sex is another interesting but understudied parameter. While many samples were mixed or consisted of women, only two studies exclusively recruited males. In view of the hormonal differences (i.e. the higher testosterone levels in men), it can be assumed that particularly strength gains will be different depending on the sex of the participants ${ }^{46}$.

\section{Conclusions}

HIFT represents an effective method to increase muscle strength and endurance capacity. In view of the multidimensional adaptations and the relatively small time effort, it may, therefore, be of value for both active and inactive individuals. Despite these promising findings, the factors moderating the treatment response remain largely obscure. In this context, future studies may specifically focus on the potential roles of age and sex. 


\begin{tabular}{|c|c|c|c|c|}
\hline Comparison & Moderator & No. of studies (ES) & Mean estimate $(95 \% \mathrm{CI})$ & Tau $^{2} /$ omega $^{2}$ \\
\hline \multicolumn{5}{|l|}{ Endurance } \\
\hline \multirow{12}{*}{ HIFT vs. NEX } & Sex & & & $0.11 / 0$ \\
\hline & Mixed & $5(10)$ & & \\
\hline & Female & $3(6)$ & $-0.88(0.19 \text { to } 1.56)^{\star}$ & \\
\hline & Intervention duration (weeks) & & & $0.28 / 0$ \\
\hline & Short $(<6)$ & $4(11)$ & & \\
\hline & Long $(>6)$ & $4(7)$ & $-0.05(-0.82$ to 0.71$)$ & \\
\hline & Session duration (min) & & & $0.28 / 0$ \\
\hline & Short $(<7)$ & $5(15)$ & & \\
\hline & Long $(>7)$ & $3(3)$ & $-0.05(-0.82$ to 0.71$)$ & \\
\hline & Total program volume (min) & & & $0.17 / 0$ \\
\hline & Low $(<168)$ & $5(11)$ & & \\
\hline & High $(>168)$ & $4(6)$ & $-0.33(-1.07$ to 0.40$)$ & \\
\hline \multicolumn{5}{|l|}{ Strength } \\
\hline \multirow{21}{*}{ HIFT vs. NEX } & Sex & & & $0.64 / 0$ \\
\hline & Mixed & $2(17)$ & & \\
\hline & Female & $4(21)$ & $0.50(-2.17$ to 1.16$)$ & \\
\hline & Age (years) & & & $0.55 / 0$ \\
\hline & Young $(<40)$ & $5(39)$ & & \\
\hline & Old $(>40)$ & $2(5)$ & $0.21(-0.56$ to 0.97$)$ & \\
\hline & Intervention duration (weeks) & & & $0.55 / 0$ \\
\hline & Short $(<7)$ & $5(39)$ & & \\
\hline & Long $(>7)$ & $2(5)$ & $0.21(-0.56$ to 0.97$)$ & \\
\hline & Session duration (min) & & & $0.60 / 0$ \\
\hline & Short $(<15)$ & $4(26)$ & & \\
\hline & Long $(>15)$ & $4(18)$ & $-0.19(-0.74$ to 1.13$)$ & \\
\hline & Total program volume (min) & & & $0.43 / 0$ \\
\hline & Low $(<168)$ & $4(22)$ & & \\
\hline & High $(>168)$ & $4(18)$ & $-0.23(-1.38$ to 0.92$)$ & \\
\hline & Interval duration (s) & & & $0.88 / 0$ \\
\hline & Short (<20 s) & $4(31)$ & & \\
\hline & Long (>20 s) & $2(5)$ & $0.12(-1.21$ to 1.45$)$ & \\
\hline & Break duration (s) & & & $0.88 / 0$ \\
\hline & Short (<10 s) & $4(31)$ & & \\
\hline & Long $(>20 \mathrm{~s})$ & $2(5)$ & $0.12(-1.21$ to 1.45$)$ & \\
\hline
\end{tabular}

Table 3. Results of the moderator analysis. HIFT high-intensity functional training, NEX no exercise, HIIT high-intensity interval training, $M C T$ moderate continuous training, no number, ES effect size, $C I$ confidence interval. Asterisks mark statistical significance of a moderator level $(\mathrm{p}<0.05)$. 
Study

Islam et al. [40]

$\mathrm{VO}_{2} \max$

$\begin{array}{ll}0.37 & 0.28\end{array}$

$5 \mathrm{~km}$ time trial

$0.68 \quad 0.29$

Time to fatigue

$0.88 \quad 0.29$

McRae et al. [30]

Bruce test

$\begin{array}{ll}0.35 & 0.59\end{array}$

$\mathrm{VO}_{2} \max$

$\begin{array}{ll}-0.42 & 0.59\end{array}$

Cunningham test

Schaun et al. [32]

$\mathrm{VO}_{2} \max$

$0.56 \quad 0.42$

Wilke et al. [39]

Watt max

$\begin{array}{ll}-0.86 & 0.37\end{array}$

$\mathrm{VO}_{2} \max$

$\begin{array}{ll}-0.20 & 0.37\end{array}$

Pooled RE
0.11
95\%-CI

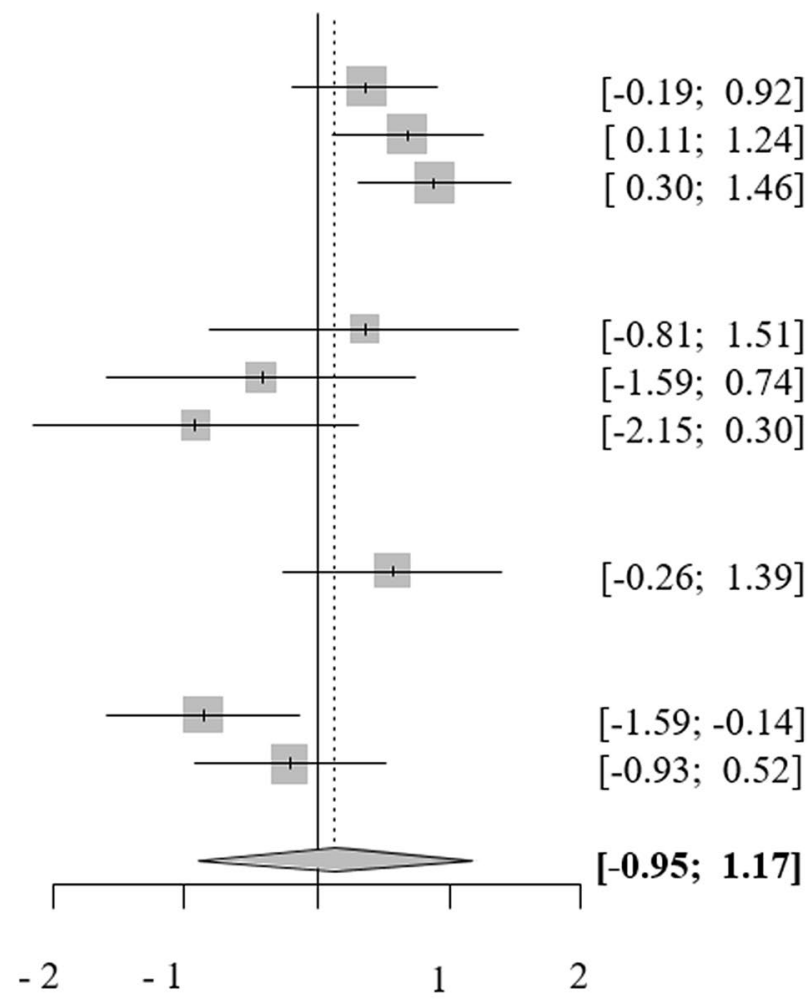

Favours HIFT

\section{Favours MCT}

Figure 5. Effects of high-intensity-functional training (HIFT) vs. moderate continuous aerobic training (MCT) on markers of endurance. Forest plots with pooled standardized mean differences (SMD), standard errors (SE) and $95 \%$ confidence intervals (CI) are displayed. $R E$ random effects.

Study

SMD SE

Buckley et al. [36]

$\mathrm{VO}_{2} \max$

Anaerobic power

Anaerobic capacity

$\begin{array}{ll}-0.18 & 0.39\end{array}$

$\begin{array}{ll}-0.06 & 0.39\end{array}$

$\begin{array}{ll}-0.14 & 0.39\end{array}$

Menz et al. [35]

$\mathrm{VO}_{2} \max$

0.560 .58

Schaun et al. [32]

$\mathrm{VO}_{2} \max$

0.84

0.43

Pooled RE

0.15
95\%-CI

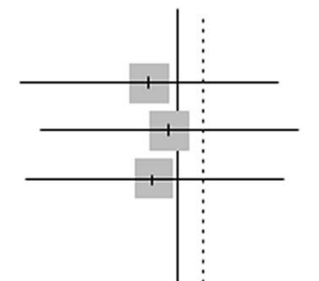

$[-0.95 ; 0.60]$

$[-0.83 ; 0.72]$

$[-0.92 ; 0.63]$

$[-0.58 ; 1.70]$

$[0.00 ; 1.69]$

$[-1.1 ; 1.4]$

\section{$\begin{array}{lllllll}-1.5 & -1 & -0.5 & 0 & 0.5 & 1 & 1.5\end{array}$}

Favours HIFT

Favours HIIT

Figure 6. Effects of high-intensity-functional training (HIFT) vs. high-intensity interval training (HIIT) on markers of endurance. Forest plots with pooled standardized mean differences (SMD), standard errors (SE) and $95 \%$ confidence intervals $(\mathrm{CI})$ are displayed. $R E$ random effects. 


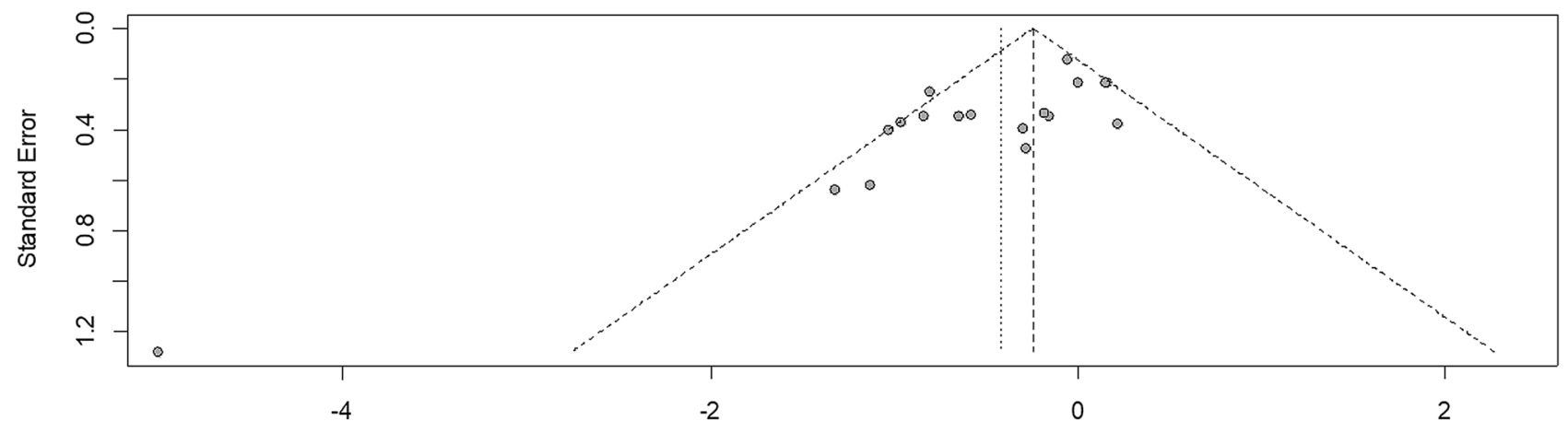

Figure 7. Funnel plot of the effect of high-intensity functional training vs. no-exercise (effect size against standard error). Note the the outlier on the lower left.

\section{Data availability}

The datasets generated during and/or analysed during the current study are available from the corresponding author on reasonable request.

Received: 25 May 2020; Accepted: 26 November 2020

Published online: 10 December 2020

\section{References}

1. Thompson, W. R. Worldwide survey of fitness trends for 2020. ACSM Health Fit J. 23, 10-18 (2019).

2. Feito, Y., Heinrich, K.M., Butcher, S.J., Poston, W,S.C. High-intensity functional training (HIFT): definition and research implications for improved fitness. Sports. https://doi.org/10.3390/sports6030076 (2018).

3. Trost, S. G., Owen, N., Bauman, A. E., Sallis, J. F. \& Brown, W. Correlates of adults' participation in physical activity: review and update. Med. Sci. Sports Exerc. 34, 1996-2001 (2002).

4. Kelly, S. et al. Barriers and facilitators to the uptake and maintenance of healthy behaviours by people at mid-life: a rapid systematic review. PLoS ONE https://doi.org/10.1371/journal.pone.0145074 (2016).

5. Wilke, J. et al. Effects of high-intensity circuit training on motor function and sport motivation in healthy, inactive adults. Scand. J. Med. Sci. Sports. 29, 144-153 (2019).

6. Heinrich, K. M., Patel, P. M., O’Leal, J. L. \& Heinrich, B. S. High-intensity compared to moderate-intensity training for exercise initiation, enjoyment, adherence, and intentions: an intervention study. BMC Pub Health. https://doi.org/10.1186/1471-2458-14789 (2014)

7. Vescovi, J. D., Murray, T. M., Fiala, K. A. \& VanHeest, J. L. Off-ice performance and draft status of elite ice hockey players. Int. J. Sports Physiol. Perform. 1, 207-221 (2006).

8. Teramoto, M., Cross, C. L., Rieger, R. H., Maak, T. G. \& Willick, S. E. Predictive validity of national basketball association draft combine on future performance. J. Strength Cond. Res. 32, 396-408 (2018).

9. Moher, D. et al. Preferred reporting items for systematic reviews and meta-analyses: the PRISMA statement. BMJ 339, b2535 (2009).

10. Wager, E. \& Wiffen, P. J. Ethical issues in preparing and publishing systematic reviews. J. Evid. Based Med. 4, 130-134 (2011).

11. Wilke, J. et al. What is evidence-based about myofascial chains: a systematic review. Arch. Phys. Med. Rehabil. 97, 454-461 (2016).

12. Krause, F. et al. Intermuscular force transmission along myofascial chains: a systematic review. J. Anat. 228, 910-918 (2016).

13. Wilke, J. et al. Acute effects of resistance exercise on cognitive function in healthy adults: a systematic review with multilevel metaanalysis. Sports Med. 49, 905-916 (2019).

14. Horsley, T., Dingwall, O. \& Sampson, M. Checking reference lists to find additional studies for systematic reviews. Cochrane Database Syst. Rev. 73, 505 (2016).

15. Rosenthal, R. Meta-analytic procedures for social research (Sage Publications, 1993).

16. Curtin, F., Altman, D. G. \& Elbourne, D. Meta-analysis combining parallel and cross-over clinical trials I: continuous outcomes. Stat. Med. 21, 2131-2144 (2002).

17. Wilke, J. et al. Acute effects of foam rolling on range of motion in healthy adults: a systematic review with multilevel meta-analysis. Sports Med. 50, 387-402 (2020).

18. Hedges, L. V., Tipton, E. \& Johnson, M. C. Robust variance estimation in meta-regression with dependent effect size estimates. Res. Synth. Methods. 1, 39-65 (2010).

19. Northey, J. M. et al. Exercise interventions for cognitive function in adults older than 50: a systematic review with meta-analysis. Br. J. Sports Med. 52, 154-160 (2018).

20. Faraone, S.V. Interpreting estimates of treatment effects. P\&T. 33, 700-703 (2008).

21. Fisher, Z., Tipton, E. Robumeta. An r package for robust variance estimation in meta-analysis. https://arxiv.org/abs/1503.02220.

22. Sterne, J.A.C, Egger, M. Regression Methods to Detect Publication and Other Bias in Meta-Analysis in Publication Bias in MetaAnalysis (eds. Rothstein, H.R., Sutton, A.J., Borenstein, M.) 99-110 (John Wiley \& Sons, 2005).

23. Maher, C. G. et al. Reliability of the PEDro scale for rating quality of randomized controlled trials. Phys. Ther. 83, 713-721 (2003).

24. Foley, C. F. et al. Estimates of quality and reliability with the physiotherapy evidence-based database scale to assess the methodology of randomized controlled trials of pharmacological and nonpharmacological interventions. Phys. Ther. 86, 817-824 (2006).

25. de Morton, N. A. The PEDro scale is a valid measure of the methodological quality of clinical trials: a demographic study. Aust. J. Physiother. 55, 129-133 (2015).

26. Engel, F. A., Rappelt, L., Held, R. \& Donath, L. Can high-intensity functional suspension training over eight weeks improve resting blood pressure and quality of life in young adults? A randomized controlled trial. Int. J. Environ. Res. Public Health. 16, 5062 (2016).

27. Engel, F. A. et al. Classroom-bades micro-sessions of functional high-intensity circuit training enhances functional strength bt not cardiorespiratory fitness in school children: a feasibility study. Front. Public Health. 7, 291 (2019).

28. Schmidt, D., Anderson, K., Graff, M. \& Strutz, V. The effect of high-intensity circuit training on physical fitness. J. Sports Med. Phys. Fitness. 56, 534-540 (2016). 
29. Ballesta-Garcia, I., Martinez-Gonzales-Moro, I., Rubio-Arias, J. A. \& Carrasco-Poyatos, M. High-intensity interval circuit training versus moderate-intensity continuous training on functional ability and body mass index in middle-aged and older women: a randomized controlled trial. Int. J. Environ. Res. Public Health. 16, 4205 (2019).

30. Sperlich, B. et al. A 4-week intervention involving mobile-based daily 6-minute micro-sessions of functional high-intensity circuit training improves strength ans qualiy of life, bit note cardio-respiratory fitness of young untrained adults. Front. Physiol. 9, 423 (2018).

31. McRae, G. et al. Extremly low volume, whole-body aerobic-resistance training improves aerobic fitness and muscular endurance in females. Appl. Physiol. Nutr. Metab. 37, 1124-1131 (2012).

32. Schaun, G. Z., Pinto, S. S., Brasil, B., Nunes, N. N. \& Alberton, C. L. Neuromuscular adaptations to sixteen weeks of whole-body high-intensity interval training compared to ergomerter-based interval and continuos training. J Sports Sci. 37, 1561-15691 (2018).

33. Schaun, G. Z., Pinto, S. S., Silva, M. R., Dolinski, D. B. \& Alberton, C. L. Whole-body high-intensity interval training induce similar cardiorespiratory adaptations compared with traditional high-intensity interval training and moderate-intensity continuous training in healthy men. J Strength Cond Res. 32, 2730-2742 (2018).

34. Evangelista, A. L., Teixeira, C. V. L. S., Machado, A. F., Pereira, P. E. \& Rica, R. Effects of a short-term of whole-body, high-intensity, intermittent training program on morphofunctional parameters. J. Bodyw. Mov. Ther. 23, 456-460 (2019).

35. Garcia-Pinillos, F., Laredo-Aguilera, J. A., Munoz-Jimenez, M. \& Latorre-Roman, P. A. Effects of 12-week concurrent high-intensity interval strength ans endurance training program on physical performance in healthy older people. J. Strength Cond. Res. 33, 1445-1452 (2019).

36. Menz, V. et al. Functional vs. running low-volume high-intensity interval training: effects on VO2max and muscular endurance. J. Sports Sci. Med. 18, 497-504 (2019).

37. Buckley, S. et al. Multimodal high-intensity interval training indreases muscle function and metabolic performance in females. Appl. Physiol. Nutr. Metab. 40, 1-6 (2015).

38. Greenlee, T. A. et al. Effectiveness of a 16-week high-intensity cardioresitance training program in adults. J. Strength Cond. Res. 31, 2528-2541 (2017).

39. Jimenez-Garcia, J. D. et al. Suspension training HIIT improves gait speed, strength ans quality of life in older adults. Int. J. Sports Med. 40, 116-124 (2019).

40. Islam, H. et al. Cardiorespiartory fitness and muscular endurance responses immediately and two-month after a whole-body Tabata or vigorous continuous training intervention. Appl. Physiol. Nutr. Metab. https://doi.org/10.1139/apnm-2019-0492 (2019).

41. Tibana, R. A. et al. Two consecutive days of crossfit training affects pro and anti-inflammatory cytokines and osteoprotegerin without impairments in muscle power. Front. Physiol. 7, 260 (2016).

42. Tibana, R. A. et al. Validity of session rating perceived exertion method for quantifying internal training load during high-intensity functional training. Sports. https://doi.org/10.3390/sports6030068 (2018).

43. Burd, N. A. et al. Muscle time under tension during resistance exercise stimulates differential muscle protein sub-fractional synthetic responses in men. J. Physiol. 590, 351-362 (2012).

44. Sterne, J. A. et al. Recommendations for examining and interpreting funnel plot asymmetry in meta-analyses of randomised controlled trials. BMJ. https://doi.org/10.1136/bmj.d4002 (2011).

45. Zwetsloot, P. P. et al. Standardized mean differences cause funnel plot distortion in publication bias assessments. Elife. https://doi. org/10.7554/eLife.24260 (2017).

46. Vingren, J. L. et al. Testosterone physiology in resistance exercise and training: the up-stream regulatory elements. Sports Med. 40, 1037-1053 (2020).

\title{
Author contributions
}

Design/concept: J.W., data collection: J.W./L.M., data analysis: J.W., drafting of the manuscript: J.W., critical revision and approval of final manuscript: J.W., L.M.

\section{Funding}

Open Access funding enabled and organized by Projekt DEAL. No sources of funding were used to assist in the preparation of this article.

\section{Competing interests}

The authors declare no competing interests.

\section{Additional information}

Correspondence and requests for materials should be addressed to J.W.

Reprints and permissions information is available at www.nature.com/reprints.

Publisher's note Springer Nature remains neutral with regard to jurisdictional claims in published maps and institutional affiliations.

\begin{abstract}
Open Access This article is licensed under a Creative Commons Attribution 4.0 International License, which permits use, sharing, adaptation, distribution and reproduction in any medium or format, as long as you give appropriate credit to the original author(s) and the source, provide a link to the Creative Commons licence, and indicate if changes were made. The images or other third party material in this article are included in the article's Creative Commons licence, unless indicated otherwise in a credit line to the material. If material is not included in the article's Creative Commons licence and your intended use is not permitted by statutory regulation or exceeds the permitted use, you will need to obtain permission directly from the copyright holder. To view a copy of this licence, visit http://creativecommons.org/licenses/by/4.0/.
\end{abstract}

(C) The Author(s) 2020 